\title{
Proposed selection strategy of surrogate matrix to quantify endogenous substances by Japan Bioanalysis Forum DG2015-15
}

\author{
Akira Wakamatsu*,1, Shoko Ochiai ${ }^{2}$, Eiko Suzuki ${ }^{3}$, Yoshinobu Yokota ${ }^{4}$, Midori Ochiai ${ }^{5}$, \\ Yosuke Kotani ${ }^{6}$, Satomi Sasahara ${ }^{7}$, Keita Nakanaga ${ }^{8}$, Yuki Hashimoto ${ }^{9}$, Satoko Ueno ${ }^{10}$, \\ Nozomu Kato ${ }^{11}$, Satoshi Kawada ${ }^{12}$, Jun Hayakawa ${ }^{13}$, Eiichi Shimada ${ }^{14}$, Shinya Horita ${ }^{15}$ \& \\ Kazuaki Sakai ${ }^{16}$ \\ ${ }^{1}$ Bioanalysis, Immunogenicity \& Biomarkers, Pre-Clinical Development Department, GlaxoSmithKline K.K. Akasaka Intersity AIR, \\ 1-8-1 Akasaka, Minato-ku, Tokyo 107-0052, Japan \\ ${ }^{2}$ Preclinical Research Unit, Drug Research Division, Sumitomo Dainippon Pharma Co., Ltd, 3-1-98 Kasugade-naka, Konohana-ku, \\ Osaka 554-0022, Japan \\ ${ }^{3}$ Drug Metabolism and Pharmacokinetics Research Laboratories, Daiichi Sankyo Co., Ltd, 1-2-58, Hiramachi, Shinagawa-ku, Tokyo \\ 140-8710, Japan \\ ${ }^{4}$ Ligand Binding, Analytical Biology, SNBL USA, Ltd, 6605 Merrill Creek Parkway, Everett, WA 98203, USA \\ ${ }^{5}$ Development Management Department, Research \& Development Division, Towa Pharmaceutical Co., Ltd, 2-5-15, Hiyoshi-cho, \\ Moriguchi, Osaka 570-0081, Japan \\ ${ }^{6}$ Research Unit 2, Pharmacokinetics and Bioanalysis Center, Shin Nippon Biomedical Laboratories, Ltd, 16-1, Minami-Akasaka, \\ Kainan, Wakayama 642-0017, Japan \\ ${ }^{7}$ Medicines Evaluation Team, Scientific Research and Business Development Department, Towa Pharmaceutical Co., Ltd, Kyoto \\ Research Park KISTIC \#202, 134, Chudoji Minami-machi, Shimogyo-ku, Kyoto, Kyoto 600-8813, Japan \\ ${ }^{8}$ Toxicology and Pharmacokinetics Laboratories, Pharmaceutical Research Laboratories, Toray Industries, Inc., 10-1, Tebiro 6-chome, \\ Kamakura, Kanagawa 248-8555, Japan \\ ${ }^{9}$ Bioanalysis Group, Osaka Laboratory, Technical Solution Headquarters, Sumika Chemical Analysis Service, Ltd, 1-135, \\ Kasugade-Naka 3-chome, Konohana-ku, Osaka 554-0022, Japan \\ ${ }^{10}$ Advanced Analytical Sciences Group, Fundamental Technology Labs., Institute For Innovation, Ajinomoto Co., Inc. 1-1, \\ Suzuki-cho, Kawasaki-ku, Kawasaki-shi, Kanagawa 210-8681, Japan \\ ${ }^{11}$ Discovery Technology Laboratories, Sohyaku Innovative Research Division, Mitsubishi Tanabe Pharma Corporation, 2-2-50, \\ Kawagihi, Toda-shi, Saitama 335-8505, Japan \\ ${ }^{12}$ Research Unit 1, Pharmacokinetics and Bioanalysis Center, Shin Nippon Biomedical Laboratories, Ltd, 16-1, Minami-Akasaka, \\ Kainan, Wakayama 642-0017, Japan \\ ${ }^{13}$ Biomarker R\&D Department, Shionogi \& Co., Ltd, 1-1, Futaba-cho 3-chome Toyonaka, Osaka 561-0825, Japan \\ ${ }^{14}$ Pharmacokinetic Research Laboratories, Ono Pharmaceutical Co., Ltd, 17-2 Wadai Tsukuba-shi, Ibaraki 300-4247, Japan \\ ${ }^{15}$ Pharamacokinetics Research Laboratories, Translational Research Unit, R\&D Division, Kyowa Hakko Kirin Co., Ltd, 1188 , \\ Shimotogari, Nagaizumi-cho, Sunto-gun, Shizuoka 411-8731, Japan \\ ${ }^{16}$ DMPK Research Department, Teijin Institute for Bio-medical Research, Teijin Pharma Ltd 4-3-2 Asahigaoka, Hino, Tokyo \\ 191-8512, Japan \\ *Author for correspondence: Tel.: + 8134231 4601; akira.2.wakamatsu@gsk.com
}

It is important to select an appropriate surrogate matrix for preparing calibration standards and quality control samples while quantitatively assaying for endogenous substances, because a blank matrix that does not contain the endogenous substance cannot be derived from the species from which the target study samples are collected. This is because the assay results might be affected, depending on the characteristics of the analyte in the surrogate matrix. Our discussion group that participated in the Japan Bioanalysis Forum discussed the recommended selection strategies, focusing on large and small molecules in ligand binding assays and LC-MS, respectively. We established an efficient selection strategy for a surrogate matrix, with simple compositions as the first candidates stated in this article.

First draft submitted: 18 April 2018; Accepted for publication: 20 July 2018; Published online: 5 September 2018

Keywords: BMV guideline $\bullet$ endogenous substance $\bullet$ evaluation of appropriateness $\bullet$ Japan Bioanalysis Forum • LBA $\bullet$ LC-MS • quantitative analysis $\bullet$ recommended method $\bullet$ selection $\bullet$ surrogate matrix 


\section{Introduction}

During traditional bioanalysis for the development of pharmaceuticals, the matrix used to prepare calibration standards should be as similar as possible to the study samples containing analytes. In principle, when calibration standards are prepared using a blank matrix, it is necessary to simultaneously develop a bioanalytical method that is not influenced by the blank matrix. The lack of interference from the blank matrix should be sufficiently confirmed during bioanalytical method validation (BMV). However, while quantifying an endogenous substance in a biological sample, the blank matrix also contains the analyte at a level that affects the analysis. In such a case, a surrogate matrix might be used in the preparation of the calibration standards and quality control (QC) samples. The surrogate matrix is also used for bioanalysis if it is difficult to obtain the matrix from sources such as bone marrow fluid, tears, skin and tissues. Nico [1] and Richard [2] reported the use of a surrogate matrix to prepare calibration standards for the quantification of endogenous substances. An ideal surrogate matrix is close in composition to the samples and completely analyte-free but there is no limit to the extent of technology adoption/increase in cost necessary for ensuring that it is identical to the studied matrix. To develop a bioanalytical method for quantifying endogenous substances within a limited development period, it is important to discuss the strategy for selecting the surrogate matrix and evaluating its validity.

\section{Examination of the topic by discussion groups}

Discussion Groups (DG) were started at the Japan Bioanalysis Forum (JBF) from 2012 onward to discuss current issues and topics that were pertinent to Japanese researchers in the bioanalytical field. In DG2014-08, a questionnaire-based survey of Japanese bioanalysts was conducted (in October 2014) to examine the topic 'Quantitative determination of endogenous substances'. Responses were obtained from 65 individuals belonging to pharmaceutical companies and bioanalysis CROs in Japan.

As a result, it was highlighted that the selection of a surrogate matrix was based on various strategies for each company and individual. Therefore, DG2015-15 examined the recommended strategies for selection of an appropriate surrogate matrix for quantitatively assaying endogenous substances in biological samples. DG members discussed the topic with regard to the analysis of large-molecular-weight endogenous substances (proteins) by ligand-binding assays (LBAs) and that of small-molecular-weight endogenous substances and peptides by LC-MS. In recent years, there have been many reports regarding the analysis of large molecular compounds by LC-MS but they have been excluded from this discussion.

\section{Points regarding the discussion for selecting a surrogate matrix for ligand-binding assay \& LC-MS} analyses

The DG 2015-15 mainly discussed the concept of selection of a surrogate matrix and standard substances from the perspective of LBA and LC-MS. In this paper, we focus on the strategy for selecting a surrogate matrix and introduce the contents of Table 1, which were discussed in DG 2015-15. The content of this paper has resulted from a discussion within a group of authors and does not represent the official opinion of the JBF.

\section{Standard substances for quantitative analysis of endogenous substances}

A standard substance is essential for quantifying endogenous substances. Though recombinant proteins with the same amino acid sequence as the analyte are often used as standard substances in LBAs, their reactivity to critical reagents and stability in actual matrices might differ from those of the analytes (endogenous substances present in biological fluids). Therefore, it should be noted that such recombinant proteins and endogenous substances cannot be considered to be identical until their equivalence is established.

In case, a small molecular compound or peptide is used as a standard substance in LC-MS, it is considered to be identical to the analyte.

\section{Selection of a surrogate matrix}

In the quantitative analysis of endogenous substances, the ideal surrogate matrix is a matrix that does not contain analyte, whose composition is similar to that of analyzed samples; however, it is expensive and often difficult to obtain. In addition, even if a surrogate matrix with an approximately similar composition is available, the composition of the surrogate matrix is not the same as that of the study sample. For this reason, it is necessary to evaluate the validity of the selected matrix and effects of using the surrogate and actual matrices on the results. 
Table 1. Content discussed by DG2015-15 regarding the strategy for selecting an appropriate surrogate matrix for use in quantitative assays for endogenous substances.

\begin{tabular}{|c|c|c|}
\hline Parameter & LBA & LC-MS \\
\hline \multicolumn{3}{|l|}{ Standard substances } \\
\hline Standard substance to be used & Recombinant proteins (large molecules) & Synthesized chemicals (small molecules, peptides) \\
\hline Profile & $\begin{array}{l}\text { Their characteristics are different from those of the } \\
\text { analyte: } \\
\text { - Responds with critical reagents } \\
\text { - Stability in the matrix }\end{array}$ & Their characteristics are identical to those of the analyte \\
\hline \multicolumn{3}{|l|}{ Selection of surrogate matrix } \\
\hline First candidate & Protein-containing buffer & Water/buffer \\
\hline $\begin{array}{l}\text { Parameters for verifying the appropriateness of the } \\
\text { selected surrogate matrix }\end{array}$ & $\begin{array}{l}\text { Parallelism/dilution linearity } \\
\text { Selectivity } \\
\text { Accuracy/precision }\end{array}$ & Accuracy/precision selectivity \\
\hline \multicolumn{3}{|c|}{ Parameters to verify the appropriateness during sample analysis } \\
\hline Evaluation item & $\begin{array}{l}\text { Dilution linearity(in case of samples having high } \\
\text { endogenous substance concentrations) }\end{array}$ & Selectivity (for the chromatographic peak of the sample) \\
\hline
\end{tabular}

Table 2. Pros and cons of the replication and dilution approaches for selecting a surrogate matrix for ligand-binding assays.

\begin{tabular}{|c|c|c|}
\hline Approach & Replication approach & Dilution approach \\
\hline Pros & $\begin{array}{l}\text { - MRD can be reduced } \\
\text { - Stability in the matrix resembles that of an actual matrix }\end{array}$ & $\begin{array}{l}\text { - It is easy to acquire. } \\
\text { - Lot-to-lot differences are small } \\
\text { - It is possible that a diluent for MRD can also be } \\
\text { used as a surrogate matrix }\end{array}$ \\
\hline Cons & $\begin{array}{l}\text { - The correct composition of an actual matrix is unclear } \\
\text { - Lot-to-lot differences are possibly large } \\
\text { - The volume of matrix required for validation and analysis in a study might be } \\
\text { unavailable (especially with an extracted matrix or rare matrix); the matrix from } \\
\text { the same lot needs to be used in validation and the sample analysis during the } \\
\text { study }\end{array}$ & $\begin{array}{l}\text { - If the matrix effect is strong, MRD becomes } \\
\text { high (low sensitivity) }\end{array}$ \\
\hline
\end{tabular}

The DG2015-15 concluded that it was not important for the matrix to be similar to the study sample while selecting a surrogate matrix. However, the authors recommended that it was essential to confirm that the surrogate matrix did not affect the assay results due to the differences between the surrogate matrix and samples. Because of this perspective, we thought that a simple solvent with a known composition should be the first surrogate matrix candidate. The criteria to be considered while selecting a surrogate matrix to be used in LBA and LC-MS have been described in the sections 'Approach for selecting a surrogate matrix for ligand-binding assay' and 'Approach for selecting a surrogate matrix for LC-MS analysis'.

\section{Approach for selecting a surrogate matrix for ligand-binding assay}

There are two possible approaches for choosing a surrogate matrix for the LBA.

The first approach involves the preparation of a surrogate matrix, whose composition resembles that of the actual sample matrix, for replicating the matrix effects as closely as possible (replication approach). The second approach involves the dilution of the actual matrix with a surrogate matrix and reducing the levels of matrix effects to those observed with the surrogate matrix (dilution approach). This dilution approach is LBA-specific.

One of the examples of the replication approach is the use of a compatible matrix of other species or removed matrix (e.g., immuno-affinity), which results in a matrix with an enhanced similarity. In the dilution approach, one buffer has a dual role, because it acts as the surrogate matrix and dilution buffer for the minimum required dilution (MRD); the MRD dilution ratio is evaluated and adjusted to reduce the matrix effects till they are insignificant. The pros and cons of both these approaches are summarized in Table 2, and these approaches are illustrated in Figure 1. 


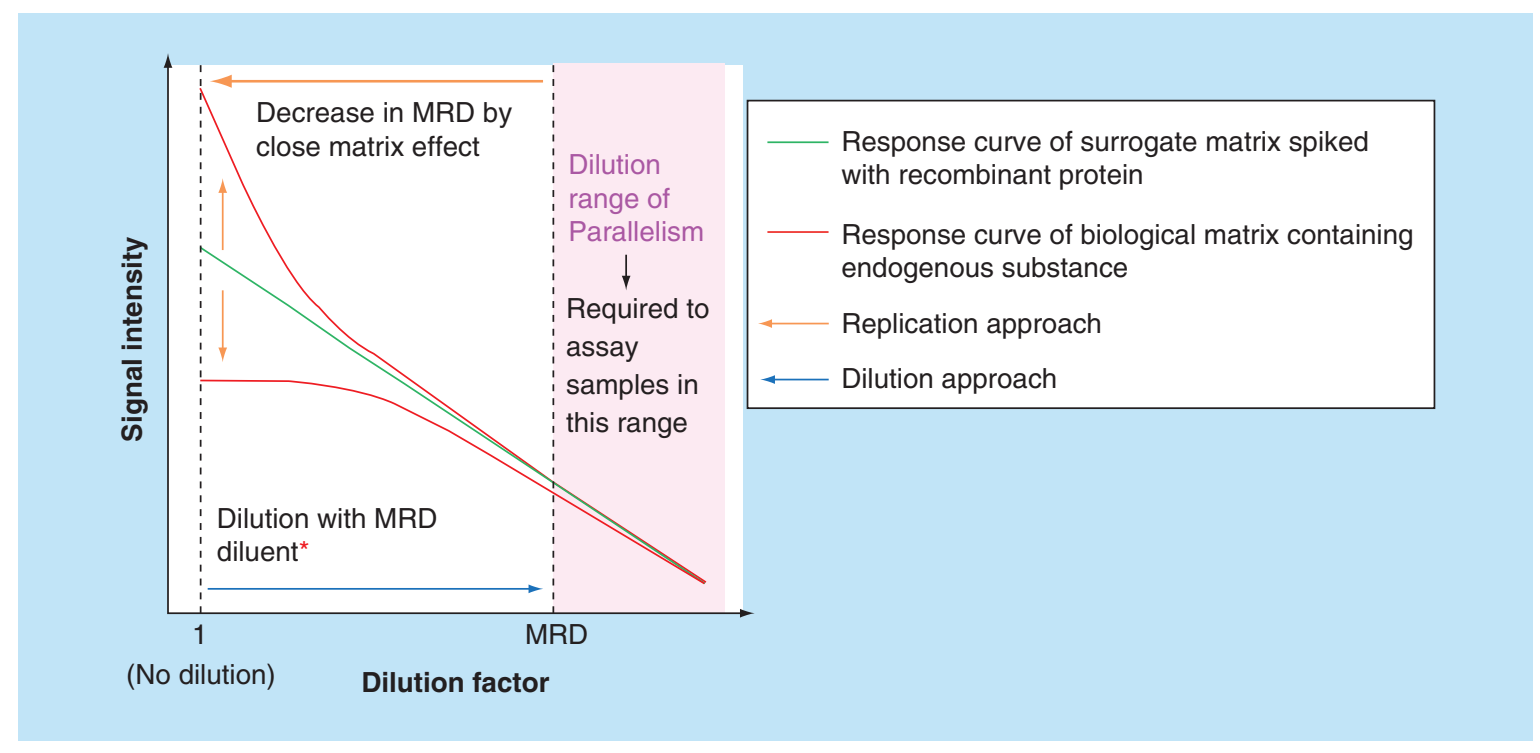

Figure 1. Concept of parallelism in ligand-binding assay and two approaches for selecting a surrogate matrix. MRD diluent is surrogate matrix in many cases in approach intended to reduce matrix effect. MRD: Minimum required dilution.

Considering the possibility of acquiring a surrogate matrix, it is easier to start method development with the 'dilution approach'. It is thought that if the validity of the analysis method could be scientifically proven, it is not essential for the composition of the surrogate matrix to resemble that of the actual matrix. Hence, the development of an analytical method using the 'dilution approach' is recommended at first. If method development using this approach is difficult, the 'replication approach' should be examined.

The following is a more detailed procedure of this selection process.

Firstly, a buffer containing a protein should be used as the first surrogate matrix candidate (and diluent for MRD, if possible) and the validity and sensitivity of the analysis method should be confirmed. When the analysis method is valid and sufficiently sensitive, the first candidate could be adopted as the surrogate matrix. If the validity and a sufficient sensitivity cannot be achieved, a re-examination of the analysis method using different buffers containing different proteins, detergents and/or chelating agents should be carried out. If none of the surrogate matrices could be adopted after using this 'dilution approach', it should be understood that this approach is unsuitable and then the 'replication approach' should be examined.

If a surrogate matrix resembling/replicating the composition of the actual matrix (actual matrices from the other species, affinity of antitarget protein antibody-extracted matrix) is used, the validity and sensitivity of the analysis method should be confirmed. When the analysis method is valid and sufficiently sensitive, the surrogate matrix can be adopted.

In cases when no surrogate matrix could be deemed acceptable, it might be necessary to change the instruments used for measurement as well as critical reagents such as antitarget antibodies. Surrogate matrix candidates are shown in Table 3. The note section of the table details their important characteristics, which require thorough consideration.

\section{Approach for selecting a surrogate matrix for LC-MS analysis}

A flowchart for the effective selection of the surrogate matrix for LC-MS analysis is shown in Figure 2.

Though it is ideal to use a surrogate matrix, which is the closest in composition to the study sample, in most cases, it is unaffordable and difficult to obtain. Even if an ideal surrogate blank matrix could be prepared, it does not match completely with the study samples; hence, it is necessary to confirm the validity as well, while selecting a simple reagent as the surrogate matrix. We conclude that similarity is not an important criterion for selecting a surrogate matrix and suggest that the essential elements of the surrogate matrix are suitable for validation criteria. In other words, it is essential to select a surrogate matrix that does not affect the quantification of study samples because 


\begin{tabular}{|c|c|c|c|c|c|}
\hline \multirow[t]{2}{*}{ Specification } & \multirow[t]{2}{*}{ Candidate } & \multicolumn{3}{|c|}{ Consideration } & \multirow[t]{2}{*}{ Note } \\
\hline & & Matrix effect & $\begin{array}{l}\text { Lot-to-lot } \\
\text { differences }\end{array}$ & $\begin{array}{l}\text { Adsorption of } \\
\text { target } \\
\text { substances }{ }^{\dagger}\end{array}$ & \\
\hline Ideal surrogate matrix & $\begin{array}{l}\text { Extracted matrix prepared } \\
\text { using an antitarget } \\
\text { substance antibody }\end{array}$ & & $\checkmark$ & & $\begin{array}{l}\text { - Preparation of an antitarget substance } \\
\text { antibody and development of an extraction } \\
\text { method would be required } \\
\text { - When the volume of matrix required is large, } \\
\text { the preparation process is complicated } \\
\text { - Expensive }\end{array}$ \\
\hline $\begin{array}{l}\text { First surrogate matrix } \\
\text { candidate }\end{array}$ & $\begin{array}{l}\text { Buffer containing a } \\
\text { protein (nonbiological } \\
\text { matrix) }\end{array}$ & $\checkmark$ & & $\checkmark$ & $\begin{array}{l}\text { - Typical protein: BSA or casein } \\
\text { - There are cases when detergents (tween, etc.), } \\
\text { chelating reagents (EDTA, etc.) and organic } \\
\text { solvents (DMSO, etc.) are added } \\
\text { - There are cases when the } \mathrm{NaCl} \text { or } \mathrm{Na}_{2} \mathrm{CO}_{3} \\
\text { concentrations require adjustment }\end{array}$ \\
\hline \multirow[t]{4}{*}{$\begin{array}{l}\text { Example for applicable } \\
\text { surrogate matrix }\end{array}$} & $\begin{array}{l}\text { Commercially available } \\
\text { matrix (include kit } \\
\text { contents) }\end{array}$ & $\checkmark$ & & $\checkmark$ & - Production may become discontinued \\
\hline & $\begin{array}{l}\text { Matrix derived from other } \\
\text { animals }\end{array}$ & $\checkmark$ & $\checkmark$ & & $\begin{array}{l}\text { - If a low concentration of endogenous } \\
\text { substances is observed in an animal, a matrix } \\
\text { derived from other animals can be used } \\
\text { - If an animal exhibits no cross reactivity to the } \\
\text { antitarget substance antibody, a matrix derived } \\
\text { from other animals can be used }\end{array}$ \\
\hline & $\begin{array}{l}\text { Matrix derived from other } \\
\text { tissues }\end{array}$ & $\checkmark$ & $\checkmark$ & & $\begin{array}{l}\text { - If a low concentration of endogenous } \\
\text { substances is observed in an organ, a matrix } \\
\text { derived from other tissues can be used }\end{array}$ \\
\hline & $\begin{array}{l}\text { Extracted matrix prepared } \\
\text { using charcoal }\end{array}$ & $\checkmark$ & $\checkmark$ & $\checkmark$ & $\begin{array}{l}\text { - Extraction efficiency is different, depending on } \\
\text { the type of endogenous substance. The } \\
\text { extraction efficiency of large molecules is lower } \\
\text { than that of small molecules } \\
\text { - Various substances containing target substances } \\
\text { can be extracted by using charcoal, after which } \\
\text { the composition of the surrogate matrix does } \\
\text { not resemble that of an actual matrix }\end{array}$ \\
\hline
\end{tabular}

its composition is different from that of actual samples. Therefore, we recommend the use of simple reagents with known components as the first surrogate matrix candidates.

One of the examples of the first surrogate matrix candidates is pure water, which is the simplest matrix and is suitable for many polar compounds. Saline and buffer solutions (e.g., phosphate buffered saline [PBS]) are also recommended. Organic solvents such as methanol or acetonitrile could also be the first surrogate matrix candidate, when analytical methods are developed for nonpolar compounds, which are expected to have a low solubility in water.

If the selected simple reagent meets the criteria for evaluation of validity, it could be employed as the surrogate matrix. Unfortunately, if the selected simple reagent could not meet the criteria, the factor (matrix effect; solubility; adsorption; stability [+ selectivity]) that affected the analysis (caused to the fail) needs to be clarified. For example, when the evaluated accuracy of the selected surrogate matrix at low analyte concentrations is not within the criteria, it is suggested that the matrix effect of this matrix is different from that of the actual matrix and/or that adsorption to the surface of tubes might be occurred. The solubility of the analyte (especially lipophilic molecules) in a simple matrix tends to be insufficient at high concentrations. Then, with reference to Table 4, another matrix should be selected as 'candidate surrogate matrix' and the selected matrix should be examined as well as the first candidate. By repeating this process, a surrogate matrix that satisfies the criteria would be selected. 


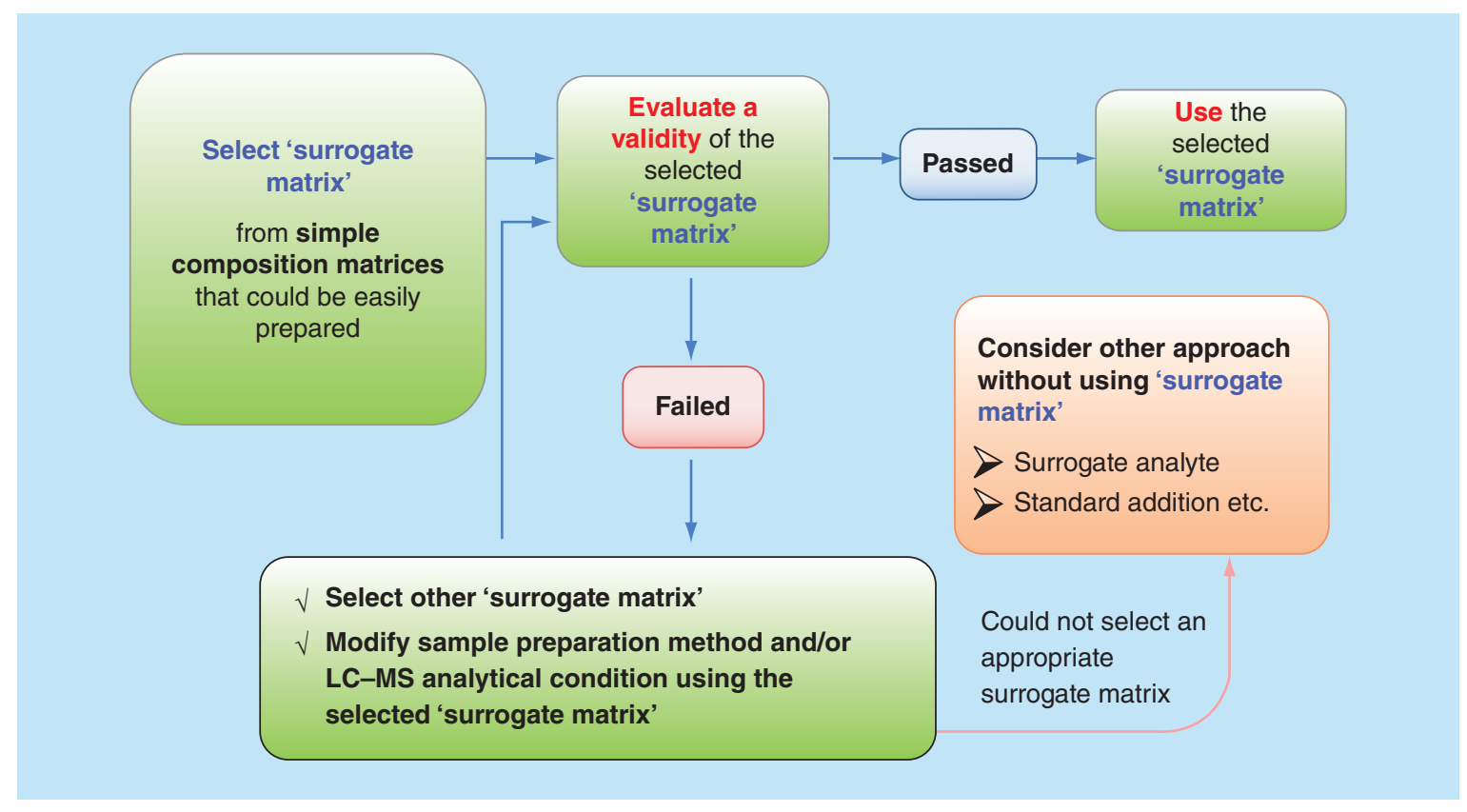

Figure 2. A flowchart for the effective selection of the surrogate matrix for LC-MS.

\begin{tabular}{|c|c|}
\hline Issue & Countermeasures \\
\hline Matrix effect & $\begin{array}{l}\checkmark \text { (A) Addition of albumin to the surrogate matrix } \\
\checkmark \text { (A) Use of commercially available surrogate matrices } \\
\checkmark \text { (B) Modification in the sample preparation methods and/or LC-MS analytical conditions }\end{array}$ \\
\hline Solubility & $\begin{array}{l}\checkmark \text { (A) Addition of albumin, organic solvent and/or surfactant to the surrogate matrix } \\
\checkmark \text { (A) Use of commercially available surrogate matrices } \\
\checkmark \text { (B) Heating (the surrogate matrix) during sample preparation }\end{array}$ \\
\hline Adsorption & $\begin{array}{l}\checkmark \text { (A) Addition of organic solvent, albumin and/or surfactant to the surrogate matrix } \\
\checkmark \text { (A) Use of commercially available surrogate matrices }\end{array}$ \\
\hline Stability & $\begin{array}{l}\checkmark \text { (A) Addition of albumin, organic solvent and/or antioxidant to the surrogate matrix } \\
\checkmark \text { (A) Adjustment of } \mathrm{pH} \\
\checkmark \text { (A) Use of commercially available surrogate matrices } \\
\checkmark \text { (B) Cooling or shading (the surrogate matrix) during sample preparation }\end{array}$ \\
\hline
\end{tabular}

As indicated above, when the first candidate of the surrogate matrix is deemed unacceptable, it is relatively easy to suggest that the root cause was the simple and well-known composition of the matrix. In addition, it is expected that an appropriate surrogate matrix could be selected in a time and cost-effective manner. Table 4 summarizes the countermeasures for each issue regarding the first surrogate matrix candidates.

For example, proteins such as albumin are added to the simple surrogate matrix to increase solubility and decrease nonspecific binding or adsorption to the hydrophobic surface of the polypropylene container. A similar effect is expected with the addition of organic solvents and surfactants. If the analyte is oxidized easily in the surrogate matrix but not in the original matrix, antioxidants such as ascorbic acid should be added to the surrogate matrix. The typical compositions of some matrices (e.g., urine, cerebrospinal fluid, serum, saliva and vaginal fluid) are already known and some synthetic matrices are commercially available. If the composition needs to be closer to that of the actual matrix, these synthetic matrices act as possible surrogate matrix candidates.

If the parameter that fails to meet the criteria is unclear or no surrogate matrix is deemed suitable, a matrix having a composition that is as similar as possible to that of the actual matrix might be needed. This approach is similar to the 'replication approach' used for matrices used in LBAs. 
The removed matrix, in which only the targeted endogenous substances are removed from the actual matrix is the analyte-free matrix that resembles the composition of the actual matrix. Affinity removal is a method for removing the target molecule selectively. Firstly, the actual matrix is applied to the affinity column, which contains the antibody against the target molecule and the flow-through is collected. In principle, only the target material(s) that the antibody can bind to are removed. However, this method is costly because a large quantity of antibodies and much time and effort might be necessary, as shown in Table 3. It is assumed that efficiency of removal of the materials is not enough due to the inhibitory effect of other endogenous substances. In that case, affinity removal might be improved by dilution with purified water. Activated charcoal is the most commonly used for the removal of endogenous substances from the matrix without using antibodies. Typically, activated carbon is added to the actual matrix and mixed for several hours, followed by the removal of activated carbon. Some commercial suppliers offer ready-to-use activated charcoal-treated serum and plasma. Importantly, activated charcoal can bind not only to the analyte, but also to other components. As a result, the composition of the activated charcoal-treated matrix might be drastically altered. Furthermore, batch-to-batch variations might occur because the level and composition of endogenous substances are different between batches; thereby, the processing time for activated carbon should be changed. Other methods such as dialysis, heating and UV irradiation could also be used to remove the analytes of interest. The matrix derived from different species of different sexes and ages, in which the analyte levels are sufficiently low, is one of the surrogate matrix candidates.

Although we did not mention them in this study, if it is difficult to select an appropriate surrogate matrix, it is necessary to switch to different methods, such as the surrogate analyte or standard addition method.

\section{Evaluation of the validity of the selected surrogate matrix}

\section{Parallelism/dilution linearity (ligand-binding assay)}

When endogenous substance concentrations in biological matrix are quantified using a calibration curve of the recombinant protein in the surrogate matrix, slopes of both concentration-response curves should be parallel. The equivalence of slopes of both curves can be evaluated by the 'parallelism' and 'dilution linearity' tests. In parallelism assessment, biological samples containing the endogenous substance are serially diluted with surrogate matrix and measured concentrations in those diluted samples are corrected with certain dilution factors. When the dilution-corrected concentrations are constant among the multiple dilutions considered, parallelism is successfully established within the applied dilution range. It should be noted that some factors possibly affect parallelism. For example, nonparallelism might be observed in the cases where a recombinant protein and an endogenous substance vary in terms of their reactivity to critical reagents and interaction with biological matrix components.

Unless the equivalence between the recombinant protein and endogenous substance is scientifically established, measured concentrations of endogenous substance are 'relative concentrations' with regard to the calibration curve of recombinant proteins, not 'absolute concentrations'. In this case, the assessment of accuracy is less meaningful. In the phase of surrogate matrix selection and validity, the equivalence between recombinant proteins and endogenous substances is usually unclear. Therefore, it appears that the determination of accuracy is not necessary for assessing parallelism.

In case of PK measurement after dosing with recombinant protein, a main component of biological samples is considered to be the recombinant protein itself, although there might be detectable endogenous substance levels in the samples as well. In that case, a parallelism-like assessment should be conducted by using a biological matrix spiked with the recombinant protein (dilution linearity). Concept of parallelism in LBA and two approaches for selecting a surrogate matrix are shown in Figure 1 (see the 'Approach for selecting a surrogate matrix for ligand-binding assay' section).

Criteria and points that need to be considered regarding parallelism/dilution linearity assessment for the quantitation of recombinant proteins and biomarkers are described in the sections 'Quantitation of recombinant protein after dosing (ligand-binding assay)' and 'Biomarker quantitation after drug treatment (ligand-binding assay)', respectively.

\section{Quantitation of recombinant protein after dosing (ligand-binding assay)}

As described above, it appears that the main component in biological samples is the administered recombinant protein. Therefore, the dilution linearity should be assessed by using a biological matrix spiked with recombinant proteins. The assessment procedure is the same as that for parallelism assessment. In this case, accuracy and precision criteria should both be set because the concentrations measured using the calibration curve of recombinant proteins 
are 'absolute concentrations'. In principle, the criteria for accuracy and precision should be in accordance with those of the BMV guideline (accuracy: within $\pm 20 \%$; precision: $\leq 20 \%$ ). If the endogenous substance concentration is above the LLOQ, the endogenous concentration should be included in calculations performed to determine accuracy (see the accuracy and precision section, 'Evaluation for accuracy and precision [LBA and LC-MS]'). It is recommended that the parallelism should be assessed in addition to dilution linearity when the endogenous concentration is high enough to assess it. It is recommended to set three or more dilution ratios for calculating precision. In principle, the precision criteria for evaluating parallelism should be the same as those set for determining the dilution linearity (equal to or less than 20\%). Ideally, the differences in parallelism should be determined by examining several lots of biological matrices.

\section{Biomarker quantitation after drug treatment (ligand-binding assay)}

It is important to assess parallelism, if the endogenous substance concentration before drug treatment is high enough. Preferably, the evaluation method used in case of PK measurement after dosing with recombinant protein should be used for the same. Precision criteria should be set case-by-case, while considering factors such as the matrix effect in biological matrices, expected change in biomarker concentration after drug treatment, perturbation of assay method and desirable assay precision. It is difficult to set reasonable precision criteria before study samples are obtained, in case of novel biomarkers, for which sufficient background information is unavailable. However, it is recommended that tentative criteria based on the BMV guideline should be set at the beginning and that these should be replaced by more reasonable criteria after obtaining sufficient information regarding study samples. Information regarding individual differences in parallelism is helpful, as seen in the case of recombinant PK. When the endogenous concentration is too low to assess parallelism, alternatively, the dilution linearity could be evaluated by using a biological matrix spiked with the recombinant protein. It should be noted that acceptable dilution linearity results do not ensure the parallelism of the endogenous substance (biomarker), because the equivalence of recombinant protein to endogenous substance is unknown in many cases. It is preferable to examine parallelism after obtaining study samples with high concentrations of endogenous substances.

\section{Slope of calibration curve (LC-MS)}

In the LC-MS method, the comparison of the slope of the calibration curve prepared using the surrogate and blank matrices might be useful for evaluating the appropriateness of the selected surrogate matrix. However, because this evaluation is not generally done by comparing the slopes of calibration curves of LC-MS, this evaluation strategy/consideration was discussed in detail (see Supplementary Data). The DG2015-15 concluded that it is unrealistic to evaluate the appropriateness of a surrogate matrix by using the slope of the calibration curve because of the following reasons:

- The reproducibility of the slope of the calibration curve is not a criterion in the BMV guideline;

- The criteria for quantitative slope evaluation is likely to be more severe than those for evaluating the QC sample accuracy (within $\pm 15 \%$ ), as shown in Supplementary Data.

Therefore, DG2015-15 recommends that the validity of the selected surrogate matrix should be evaluated using the values for accuracy and precision, as described in the next section especially for the LC-MS method and not using the slope of the calibration curve.

\section{Evaluation for accuracy \& precision (ligand-binding assay \& LC-MS)}

In order to evaluate the validity of the surrogate matrix, it is necessary to confirm the accuracy and precision in both LBA and LC-MS. We decided that four QC sample concentrations (LLOQ, LQC, MQC and HQC) should be evaluated. When preparing a QC sample with an actual matrix, the combined concentration of the endogenous substance and spiked standard substance was expected to exceed the quantification range; hence, the QC-ULOQ was excluded while carrying out the evaluation. In the case of an endogenous substance being administered as a medicine, in principle, the accuracy and precision should be evaluated according to the BMV guideline. However, during biomarker quantification, the evaluation criteria should be set case-by-case, referring to the information including the previously determined endogenous substance concentration and known information, such as literature, nonclinical information, target variance width (precision), PK guideline and so on. 


\begin{tabular}{|c|c|c|c|c|c|}
\hline \multicolumn{5}{|c|}{ Preparation of QC samples: example } & \multirow[b]{2}{*}{$\begin{array}{l}\text { Procedure } \\
\text { 1. Spike standard substance to actual matrix }\end{array}$} \\
\hline $\begin{array}{l}\text { QC } \\
\text { conc }\end{array}$ & A & B & $\mathrm{C}$ & $\mathrm{D}$ & \\
\hline HQC & 1 & 1 & 1 & 4 & $\begin{array}{l}\text { 2. Spike standard substance to } \\
\text { surrogate/removed matrix }\end{array}$ \\
\hline MQC & 1 & 1 & 1 & - & $\begin{array}{l}\text { 3. Spike standard substance to diluted actual } \\
\text { matrix with surrogate matrix to be low level } \\
\text { of endogenous substance }\end{array}$ \\
\hline LQC & 1 & 2 & 3 & 4 & 4. Diluted actual matrix with surrogate matrix \\
\hline LLOQ & 1 & 2 & 3 & - & spike standard substance) \\
\hline \multicolumn{6}{|c|}{ Endogenous levels in actual matrix } \\
\hline \multicolumn{6}{|c|}{$\begin{array}{l}<\text { Endogenous concentration between } Q C \text { samples and actual matrix }> \\
A<L L O Q<L Q C<M Q C<H Q C \\
L L O Q<L Q C<B, C<M Q C<H Q C \\
L L O Q<L Q C<M Q C<H Q C<D \text { (in biomarker for } L B A)\end{array}$} \\
\hline
\end{tabular}

Figure 3. Example of the preparation of quality control samples.

The following two equations are proposed to calculate the accuracy during the quantitation of endogenous substances:

1. (Equation 1) accuracy $(\%)=$ determined concentration of QC sample / (endogenous concentration + spiked concentration)

2. (Equation 2) accuracy (\%) = (determined concentration of QC sample - endogenous concentration) / spiked concentration

The features of each of these are as follows:

- (Equation 1) Evaluating the accuracy at a concentration that is the sum of the spiked and endogenous concentration: if the spiked concentration is lower than that of the endogenous substance concentration, the accuracy values are occasionally within the acceptance criteria, even if the spiked concentration cannot be quantified precisely. Therefore, the criteria should be predefined, considering the balance between the endogenous substance concentration and spiked concentration.

- (Equation 2) Evaluating the accuracy at the spiked concentration of standard solution. When the spiked concentration is lower than the endogenous substance concentration, it would be difficult for the accuracy values to be within the acceptance criteria, rather than that calculated using Equation 1.

\section{Preparation of quality control samples}

It is necessary to consider the method for preparing the QC samples, based on the concentrations of QC sample to be prepared and endogenous substance concentration in the actual matrix. The matrix to be used would be selected after considering the QC sample concentration to be prepared. An example of the method for QC sample preparation is summarized in Figure 3.

When the concentration of the endogenous substance in the actual matrix is very low (i.e., lower than the LLOQ), the standard substance would be added to the actual matrix to achieve QC samples with desired concentrations (LLQC, LQC, MQC and HQC) in the same manner as the traditional quantification of the exogenous substance (Figure 3A).

If the concentration of QC samples to be prepared is lower than that of the endogenous substance in the actual matrix, the blank matrix diluted with a surrogate matrix, removed matrix or surrogate matrix should be used to prepare the QC sample. A QC sample is prepared by using one of these matrices and adding a standard substance 
or endogenous substance (Figure 3B, Figure 3C - LLOQ, LQC). It is recommended to use the actual matrix for the preparation of $\mathrm{QC}$ samples with concentrations that are higher than those of the endogenous substance in the actual matrix (Figure 3B, Figure 3C - MQC, HQC).

In addition, QC samples could be prepared by diluting the actual matrix with a surrogate matrix within the concentration range where dilution parallelism is confirmed, in some cases of LBA biomarker quantification (Figure 3D). When preparing the QC sample, the actual matrix should preferably be a pooled matrix.

\section{Evaluation of selectivity (ligand-binding assay \& LC-MS)}

The concept of selectivity evaluation differs between LBA and LC-MS, confirmation of matrix effect in LBA and confirmation of presence or absence of interference peaks in LC-MS. The selectivity evaluation of the LBA and LC-MS methods are described in the sections 'Selectivity (ligand-binding assay) in recombinant PK' and 'Selectivity (LC-MS/MS)' respectively.

\section{Selectivity (ligand-binding assay) in recombinant PK}

The selectivity in the biological matrix should be confirmed in case of recombinant PK, in a manner similar to that done for fulfilling the general requirements of bioanalytical methods for a drug candidate. Selectivity samples are prepared by spiking a recombinant protein into a biological matrix or endogenous substance-removed matrix. The endogenous concentration should be included in calculations to determine accuracy (see the accuracy and precision section 'Evaluation for accuracy and precision (LBA and LC-MS)'. Acceptance criteria and test sample numbers should be set in accordance with the BMV guideline.

\section{Selectivity (LC-MS/MS)}

Selectivity evaluation of the surrogate matrix in LC-MS/MS

In the surrogate matrix, it should be confirmed that an interference peak is absent at the retention time of the analyte and internal standard. In the case of the matrices derived from different animal species and other tissue and the matrix in which analyte was removed from actual matrix by using an antianalyte antibody, selectivity should be evaluated with at least six lots of matrices. In the case that a nonbiological matrix (such as water, buffer, artificial CSF and synthetic urine) is used, one lot of sample should be enough, because they do not contain an analyte. The acceptance criteria should be in accordance with those of the BMV guidelines and either an endogenous peak should not be found in the blank sample matrix or the peak area of the endogenous peak should be less than $20 \%$ of the analyte peak area at the LLOQ and 5\% of the internal standard peak area.

Evaluation of selectivity in actual matrix in LC-MS/MS

To ensure that the endogenous substance concentration measured by LC-MS/MS in the actual sample is approximately correct, it is necessary to confirm that the accuracy and precision of QC samples are within the required levels and that there is no problem regarding selectivity in the actual matrix. An example of a method for evaluating selectivity in the actual matrix is shown below:

(M1) The standard substances and actual matrix are evaluated using an established method, with multiple (three or more) transitions for each analyte. The ratio of the peak area of each transition to the peak area of the transition used for quantification in the established method is calculated; the results should confirm that the ratios are the same between the standard substance and sample.

(M2) The sample is measured by using a method in which a mobile phase, a gradient or a column is changed in the established assay conditions. The conditions are changed so that the substance can be separated from related substances and contaminants; the separation achieved with this method is different from that observed with the previous method. When LC conditions are changed, the retention time of the peak observed in the actual sample should be the same as that of the standard substance and it should be confirmed that it is not divided into multiple peaks.

(M3) If isomers of the analyte are present and standard substances constituting the isomers are available, it should be confirmed that the retention time of the analyte and the isomer are different in the established method.

In order to evaluate the selectivity more accurately, it is desirable to implement (M1) (execute [M3] if necessary). If it is difficult to implement (M1), in cases such as when multiple product ions are not generated, (M2) should be selected. Other evaluation methods described below are also options, but it should be noted that each method is challenging. 
(M4) When standard substance and sample concentrations are measured with a high-resolution mass spectrometer, it should be confirmed that the precise mass of the peak observed in the actual sample matches that of the standard substance. However, even if the peak observed in the actual sample was an isomer of the substance, it could not be distinguished.

(M5) When standard substance and samples containing the analyte are derivatized, it should be confirmed that the retention time of the peak observed in the actual sample is consistent with that of the standard substance. However, if the endogenous peak observed in the sample has the same functional group as that of the analyte, the same behavior is observed even if these substances are nonidentical.

(M6) It is also possible to confirm whether the quantitative value calculated by LC-MS conforms to the quantitative value calculated by other methods, such as LC-UV, LC-FLR or LBA. However, the results should be judged while considering the nature of the substance and the principle of the analytical method used for the comparison.

\section{Conclusion}

This article has presented the topics discussed by the DG 2015-15 regarding:

- The recommended strategies for selecting a surrogate matrix, focusing on large molecules in LBA and small molecules in LC-MS for the quantitative analysis of endogenous substances in biological samples;

- Evaluation of the validity of the selected surrogate matrix and;

- QC sample preparation according to anticipated endogenous substance concentrations.

The group reached a consensus that the similarity in the composition of the matrix was not essential, as long as the required accuracy/precision (for both LBA and LC-MS), parallelism/dilution linearity and selectivity (for LBA) were achieved in quantitation. Based on that, we established an efficient strategy for selecting a surrogate matrix from the following matrices with simple compositions, which were used as the first candidates:

- LBA (for large molecules); protein (e.g., BSA, casein) containing buffer;

- LC-MS (for small molecules); simple solvent (e.g., water, buffer).

On the other hand, in two Japanese BMV guidelines and their Q \& As [3-6], EMA guidelines [7] and the US FDA guidance [8], it has been stated that "the use of a surrogate matrix should be justified as much as possible in the course of establishing the analytical method," regarding the surrogate matrix used for preparation of standard and QC samples. The selection and verification of surrogate matrices are important from a regulatory perspective. It is fortunate that this proposal would help in the quantitative analysis of endogenous substances in biological samples.

\section{Disclaimer}

The discussion and conclusions are the results of voluntary discussion within the discussion group and are not the authorized opinions of the JBF or Japanese regulatory authorities.

\section{Acknowledgements}

The authors thank A Iwata, Takeda-Teva Pharma Ltd, the steering committee of the Japanese Bioanalysis Forum and the participants of the survey regarding the quantitative analysis of endogenous substances in 2014 and the attendees of the 6th (2015 DG 2014-08) and 7th (2016 DG 2015-15) Japan Bioanalysis Forum symposium who participated in a discussion during the poster session of the symposium. We also wish to thank T Osumi, Otsuka Pharmaceutical Co., Ltd, C Kitajima, LSI Medience Corporation and T Yamaguchi, Sumika Chemical Analysis Service, Ltd for discussing and advising about the survey, along with the members participating in the DG 2014-08 and the 6th JBF Symposium.

Financial \& competing interests disclosure

A Wakamatsu is an employee of GlaxoSmithKline KK. S Ueno is an employee of Ajinomoto Co. Inc. S Ochiai is an employee of Sumitomo Dainippon Pharma Co. Ltd. M Ochiai and S Sasahara are employees of Towa Pharmaceutical Co. Ltd. N Kato is an employee of Mitsubishi Tanabe Pharma Corporation. S Kawada and Y Kotani are employees of Shin Nippon Biomedical Laboratories Ltd. E Shimada is an employee of Ono Pharmaceutical Co. Ltd. E Suzuki is an employee of Daiichi Sankyo Co. Ltd. K Nakanaga is an employee of Toray Industries Inc. Y Hashimoto is an employee of Sumika Chemical Analysis Service Ltd. J Hayakawa is an employee of Shionogi \& Co. Ltd. S Horita is an employee of Kyowa Hakko Kirin Co. Ltd. Y Yokota is an employee of SNBL USA Ltd. K Sakai is an employee of Teijin Pharma Ltd. The authors have no other relevant affiliations or financial involvement with any organization 
or entity with a financial interest in or financial conflict with the subject matter or materials discussed in the manuscript apart from those disclosed. This includes employment, consultancies, honoraria, stock ownership or options, expert testimony, grants or patents recieved or pending, or royalties.

This article has been edited and proofread by a professional scientific editing company, Editage.

\section{Author contributions}

All the authors were members of DG2015-15 and equally contributed to discussion. K Sakai, S Horita, Y Yokota and E Shimada lead the discussion. S Ochiai, E Suzuki, Y Yokota, M Ochiai, Y Kotani, S Sasahara, K Nakanaga, Y Hashismoto, S Ueno, N Kato, S Kawada, J Hayakawa wrote the draft manuscript and A Wakamatsu compiled and revised it. All the authors reviewed and approved the final manuscript.

\section{Open access}

This work is licensed under the Attribution-NonCommercial-NoDerivatives 4.0 Unported License. To view a copy of this license, visit http://creativecommons.org/licenses/by-nc-nd/4.0/

\section{References}

1. van de Merbel NC. Quantitative determination of endogenous compounds in biological samples using chromatographic techniques. Trends Analyt. Chem. 27(10), 924-933 (2008).

2. Houghton R, Pita CH, Ward I, Macarthur R. Generic approach to validation of small-molecule LC-MS/MS biomarker assays. Bioanalysis 1(8), 1365-1374 (2009).

3. MHLW. Guideline on Bioanalytical Method Validation in Pharmaceutical Development. PFSB/ELD Notification No. 0711-1 dated July 11 (2013). http://www.pmda.go.jp/files/000206209.pdf

4. MHLW. Guideline on Bioanalytical Method (Ligand Binding Assay) Validation in Pharmaceutical Development. PFSB/ELD Notification No. 0401-1 dated April 11 (2013). http://www.pmda.go.jp/files/000206208.pdf

5. MHLW. Questions and Answers (QنA) for the Guideline on Bioanalytical Method Validation in Pharmaceutical Development. Office Communication dated July 11 (2013). http://www.pmda.go.jp/files/000206209.pdf

6. MHLW. Questions and Answers (QUA) for the Guideline on Bioanalytical Method (Ligand Binding Assay) Validation in Pharmaceutical Development. Office Communication dated April 1 (2014). http://www.pmda.go.jp/files/000206208.pdf

7. EMA. Guideline on Bioanalytical Method Validation (2011). http://www.ema.europa.eu/ema/pages/includes/document/open_document.jsp?webContentId=WC500109686

8. US FDA. Guidance for Industry, Bioanalytical Method Validation (2018). https://www.fda.gov/downloads/Drugs/GuidanceComplianceRegulatoryInformation/Guidances/UCM070107.pdf 